\title{
Substituição isolada da valva aórtica em pacientes com função ventricular deprimida
}

\author{
Gilberto Venossi BARBOSA*, Blau F. de SOUZA**, Nei A. REY**, Orlando Carlos WENDER**, Pedro \\ PIMENTEL FILHO**, Sívia Regina Rios VIEIRA*, Hilário WOLMEISTER*, Waldomiro C. MANFROI***, Paulo \\ P. WESTPHALEN*
}

RBCCV 44205-93

BARBOSA, G. V.; SOUZA, B. F.; REY, N. A.; WENDER, O. C.; PIMENTEL FILHO, P.; VIEIRA, S. R. R.; WOLMEISTER, H.; MANFROI, W. C.; WESTPHALEN, P. P. - Substituição isolada da valva aórtica em pacientes com função ventricular deprimida. Rev. Bras. Cir. Cardiovasc., 4(3): 195-201, 1989.

RESUMO: Para avaliar o valor prognóstico da fração de ejeção do ventrículo esquerdo, entre 210 pacientes com lesōes da valva aórtica operados, consecutivamente, entre maio de 1981 e outubro de 1988 e que receberam as próteses Omniscience e Meditronic-Hall, foram selecionados 112 e divididos, de acordo com a fraçăo de ejeçăo do ventrículo esquerdo, em dois grupos: o G1 $=\mathrm{FE}>40 \%$, ficou com 52 pacientes com médias de idade $39 \pm 12$ anos, $\mathrm{FE}=58 \pm 10 \%$ e classe funcional (NYHA) $=2,8 ; \circ \mathrm{GE}=\mathrm{FE} \leqq 40 \%$ ficou com 60 pacientes com médias de idade $48 \pm 17$ anos, $\mathrm{FE}=27 \pm$ $13 \%$ e $($ NYHA $)=3,6$. Nas cirurgias, foram utilizados hipotermia e hemodiluição moderadas, oxigenador de bolhas, infusão cardioplégica St. Thomas. As médias dos tempos de circulação extracorpórea e parada cardiaca foram: no G1 = $82 \pm 18$ e $49 \pm 7$ minutos, e $96 \pm 11$ e $55 \pm 6$ minutos, no Grupo 2; o tamanho das próteses foi $25,2 \pm 1,8$ milimetros. No Grupo 1, a mortalidade imediata foi $3,8 \%$ no G1 e 5,8 no G2, e a tardia $4 \%$ no G1, e no G2 foi $8,7 \%$. No G1 houve significante associaçāo entre a mortalidade e a função ventricular sistólica. Clínicamente, no G1, $65 \%$ dos pacientes estão na (NYHA) I, $28 \%$ na II, $5 \%$ na III e $2 \%$ na IV. No G2, $46 \%$ estão na (NYHA) I, $25 \%$ na II, $13 \%$ na III, e $16 \%$ na IV. A sobrevida atuarial em sete anos foi $88 \pm 2 \%$ no G1 e $76 \pm 4 \%$ no G2. Portanto, os pacientes com FE $\leqq 40 \%$ apresentaram mortalidade mais elevada, menor sobrevida a curto e longo prazo e resultados clínicos menos satisfatórios. Para obtermos melhores resultados, devemos operar antes que a FE em repouso e exercício se torne deprimida, ou que apareça intolerância ao exercício moderado.

DESCRITORES: função ventricular; valva aórtica, cirurgia.

\section{INTRODUÇĀO}

A substituição valvar tem sido indicada nos pacientes com lesōes da valva aórtica e severamente sintomáticos e se constitui no maior avanço das últimas três décadas.
A utilização de novas próteses, entre as quais as de disco basculante, aliadas aos avanços na avaliação pré-operatória, na técnica cirúrgica, na preservação do miocárdio e nos cuidados pós-operatórios tornaram este método de terapêutica bastante seguro ${ }^{1,9,32}$. Apesar disto, persiste uma mortalidade operatória de 3 a $10 \%$,

Trabalho realizado nos Serviços de Cirurgia Cardiovascular do Hospital de Clínicas de Porto Alegre e Hospital Nossa Senhora da Conceiçăo, Porto Alegre, RS, Brasil.

Apresentado ao 16: Congresso Nacional de Cirurgia Cardiaca. São Paulo, 7 e 8 de abril, 1989.

- Do Hospital de Clínicas de Porto Alegre e Hospital Nossa Senhora da Conceiçăo.

* Do Hospital Nossa Senhora da Conceição.

*.. Do Hospital de Clínicas de Porto Alegre.

Endereço para separatas: Gilberto Venossi Barbosa. Rua Saul Monnemacher, 52. 91700 Porto Alegre, RS, Brasil. 
BARBOSA, G. V.; SOUZA, B. F.; REY, N. A.; WENDER, O. C.; PIMENTEL FILHO, P.; VIEIRA, S. R. R.; WOLMEISTER, H.; MANFROI, W. C.; WESTPHALEN, P. P. - Substituição isolada da valva aórtica em pacientes com função ventricular deprimida.

Rev. Bras. Cir. Cardiovasc., 4(3): 195-201, 1989.

ou mais, relacionada às condiçōes do miocárdio ventricular, e uma sobrevida a longo prazo menor que a da população da mesma faixa etária e sexo ${ }^{28,31}$. Alguns pacientes continuam com sintomas limitantes após a operação, mesmo com bom desempenho da prótese ${ }^{6-8}$.

Estes fatos levaram GAULT et alii ${ }^{17}$ e HILDNER et alii ${ }^{21}$ a afirmarem que a diminuição da função ventricular esquerda impossibilita qualquer melhora no desempenho miocárdico, após a operaçāo. Existem vários relatos, na literatura, em apoio a esta afirmativa ${ }^{14}, 16,17$, e outros demonstrando o contrário ${ }^{4,6-8}$, acentuando esta controvérsia.

O objetivo deste trabalho é avaliar o valor prognóstico da fração de ejeção do ventrículo esquerdo na mortalidade imediata, sobrevivência e melhora clínica tardia, numa série clínica com o intuito de indicar o momento oportuno da cirurgia.

\section{CASUISTICA E MÉTODOS}

No período de maio de 1981 a outubro de 1988 , 201 pacientes consecutivos com lesōes da valva aórtica foram operados, sendo $76 \mathrm{com}$ insuficiência (IAo), 67 com estenose (EAo) e 58 com dupla lesão (DLAo). Destes, 113 receberam a prótese Omniscience (OMS) e 88 a prótese Meditronic-Hall (MH). Excluímos, então, os pacientes que tiveram outros procedimentos cirúrgicos associados, os que receberam próteses de tamanho menor que 23 , os que possuiam estudo cineangiocardiográfico de má qualidade, impossibilitando o cálculo da fração de ejeção e aqueles sem seguimento clínico completo.

Ficamos, então, com 112 pacientes com lesão isolada da valva aórtica, os quais foram divididos de acordo com a fração de ejeção do ventrículo esquerdo, em dois grupos: G1 - FE > $40 \%$ e GE - FE $\leqslant 40 \%$, cujas características pré-operatórias estão expostas na Tabela 1.

Os dados foram coletados dos prontuários dos pacientes e atualizados nas visitas ambulatoriais, ou por contato com os médicos assistentes.

TABELA 1

CARACTERISTICAS DOS GRUPOS EM ESTUDO

\begin{tabular}{|c|c|c|c|c|c|c|c|}
\hline \multirow{2}{*}{ Grupos } & \multirow{2}{*}{$\begin{array}{l}N \cdot \\
p\end{array}$} & \multicolumn{2}{|c|}{ Sexo } & \multicolumn{2}{|c|}{ Idade } & FEVE & \multirow{2}{*}{$\begin{array}{c}N Y H A \\
\bar{x}\end{array}$} \\
\hline & & $m$ & $f$ & $\bar{x}$ & $D P$ & $D P$ & \\
\hline $\mathrm{G} 1-\mathrm{FE}>40 \%$ & 52 & 37 & 15 & 39 & \pm 12 & $58 \pm 10$ & 2,8 \\
\hline $\mathrm{G} 2-\mathrm{FE} \leqslant 40 \%$ & 60 & 41 & 19 & 48 & \pm 17 & $27 \pm 13$ & 3,6 \\
\hline
\end{tabular}

\section{Obtenção da Fração de Ejeção}

Os filmes das cineventriculografias esquerdas foram projetados na pantalha do projetor Tagarno e, entre os múltiplos contornos diastólicos e sistólicos finais bem delimitados, na ausência de extra-sístoles, foram selecionados dois deles obtidos em seqüência. Sobre a pantaIha do projetor, foi acoplada uma folha branca de papel ofício, na qual foram delineados os contornos do VE, ao final da diástole e da sístole.

Para o cálculo da (FE) foram utilizados o volume diastólico final (VDF) e o volume sistólico (VSF), conceituados por DODGE et alii ${ }^{13}$ e modificados por GREENE et alii ${ }^{19}$, KENNEDY et alii ${ }^{23}$, para cineventriculografia em um pulmão. A fórmula condensada volume ventricular $=0,81\left(8 / 3 \times \mathrm{A}^{2} / \mathrm{L} \times \mathrm{FC}^{2}\right)+1,9$, na qual 0,81 e 1,9 são constantes obtidas da equação regressiva, aplicadas para a projeção em obliqua anterior direita (OAD) em um plano.

- 0,81 e $8 / 3=$ constantes obtidas da fórmula da equação para o cálculo do biplano.

- $\mathrm{A}^{2}$ = área do (VE), no final da diástole ou sístole obtida por planimetria.

- $\mathrm{L}, \mathrm{e}=$ eixo longitudinal do (VE) no final da diástole e na sistole.

- $F C^{2}=$ fatores de correção da magnificação radiológica.

Uma vez obtidos os volumes, resolveu-se a fórmula $\mathrm{FE}=\frac{\mathrm{VDF}-\mathrm{VSF}}{\mathrm{VDF}}$; estes dados foram processados eletronicamente, num equipamento HP - 9810-A, com um sistema de programação para cálculo da função cardiaca. Consideramos normal uma FE $=67 \%$ e deprimida inferior a $40 \%$.

As cirurgias foram realizadas utilizando-se oxigenador de bolhas descartável, hemodiluição e hipotermia sistêmica moderadas de 28 a $30^{\circ} \mathrm{C}$ e com fluxo de perfusão de 2,2 litros por minuto por metro quadrado de superfície corporal. O método de proteção miocárdica constituiu-se na infusāo direta de solução cardioplégica nos óstios coronarianos, tipo St. Thomas, a $4^{\circ} \mathrm{C}$ e do banho intrapericárdico com Ringer lactado gelado. A fixação das próteses no anel valvar foi feita com pontos em dupla laçada com fio 2-0 de Poliester arterial. A abertura maior da prótese foi orientada para o seio coronariano direito com a finalidade de maximizar o fluxo com menor turbulência. As variáveis trans-operatórias nos dois grupos estão na Tabela 2.

\section{MÉTODO ESTATÍSTICO}

Cálculo das porcentagens para a mortalidade imediata e classificação funcional pós-operatória. Cálculo de probabilidade de sobrevivência a longo prazo, pelo método atuarial modificado por GRUNKMEIER \& 
BARBOSA, G. V.; SOUZA, B. F.; REY, N. A.; WENDER, O. C.; PIMENTEL FILHO, P.; VIEIRA, S. R. R.; WOLMEISTER, H.; MANFROI, W. C.; WESTPHALEN, P. P. - Substituição isolada da valva aórtica em pacientes com função ventricular deprimida.

Rev. Bras. Cir. Cardiovasc., 4(3): 195-201, 1989.

TABELA 2

MÉDIAS DAS VARIÁVEIS TRANS-OPERATÓRIAS

\begin{tabular}{cccc}
\hline Grupos & $\frac{T C E(m i n)}{\bar{X} D P}$ & $\frac{T P C(m i n)}{X D P}$ & $\frac{T P R(m m)}{\bar{X} D P}$ \\
\hline G1 FE $>40 \%$ & $82 \pm 18$ & $49 \pm 7$ & $25,2 \pm 1,8$ \\
GS $\mathrm{NE} \leqslant 40 \%$ & $97 \pm 11$ & $55 \pm 6$ & $25,6 \pm 1,4$
\end{tabular}

TCE $=$ tempo de circulaçăo extracorpórea; TPC = tempo de parada cardiaca; TPR $=$ tamanho da prótese .

TABELA 3

MORTALIDADE

\begin{tabular}{lcccc}
\hline \multirow{2}{*}{ Grupos } & \multicolumn{2}{c}{ Imediata } & \multicolumn{2}{c}{ Tardia } \\
& $N^{*}$ & $\%$ & $N^{0}$ & $\%$ \\
\hline G1 FE $>40 \%$ & 2 & 3,8 & 2 & 4,0 \\
G2 FE $\leqslant 40 \%$ & 3 & 5,0 & 5 & 8,7 \\
\hline
\end{tabular}

STARR $^{20}$. A comparação entre as médias dos grupos G1 e G2 e respectivos niveis de significância pelo teste de Qui-quadrado $\left(\boldsymbol{\gamma}^{2}\right)$, pertencente a um pacote estatístico Epistat, processado em microcomputador Craft-XT. Foi adotado o nível de significância de 0,05.

\section{RESULTADOS}

Analisando os dados da Tabela 1, vemos que os pacientes do Grupo G2 são mais idosos, com uma diferença de nove anos, na média, para o Grupo G1. Apresentam-se em classe funcional mais avançada, concursando entre a NYHA III e IV; a média da FE é menos da metade, comparada à de G1.

As variáveis trans-operatórias tiveram seus valores aproximados entre os dois grupos e não apresentaram significância (NS).

A mortalidade imediata de 0 a 30 dias e a tardia estão expostas na Tabela 3. Mostraram-se aproximadas (NS); o mesmo não ocorreu com a tardia, que, no Grupo G2, foi o dobro do G1.

No pós-operatório imediato, três pacientes do grupo G1 (5\%) necessitaram suporte inotrópico endovenoso por sinais de baixo débito transitório com padrão de isquemia subendocárdica. Dois tiveram recuperação sem seqüelas e um evoluiu para infarto do miocárdio (2\%) e, mais tarde, para NYHA IV. No Grupo G2, 12 pacientes (20\%) necessitaram de drogas inotrópicas para tratamento da síndrome de baixo débito. Três (5\%) tiveram óbito, um dos quais por taquicardia ventricular e fibrilação recurrente refratária. Dos restantes, três mostraram padrăo de infarto subendocárdico e um transmural $(6,6 \%)$, evoluindo para a NYHA III e IV, respectivamente. Houve,
TABELA 4

CAUSAS DOS OBITOS IMEDIATOS

\begin{tabular}{|c|c|c|}
\hline \multirow{2}{*}{ Causas } & \multicolumn{2}{|c|}{ Grupos } \\
\hline & $\overline{G 1}$ & $\overline{G 2}$ \\
\hline Síndrome de baixo débito & 一 & 2 \\
\hline Arritmia ventricular & 一 & 1 \\
\hline Coagulopatia pós-perfusão & 1 & - \\
\hline Insuficiência renal aguda & 1 & - \\
\hline TOTAL & $2(3,8 \%)$ & $3(5,0 \%)$ \\
\hline
\end{tabular}

TABELA 5

CAUSAS DOS OBITOS TARDIOS

\begin{tabular}{lcc}
\hline \multicolumn{1}{c}{ Causas } & \multicolumn{2}{c}{ Grupos } \\
\cline { 2 - 3 } & \multicolumn{1}{c}{ G1 } & G2 \\
Insuficiência cardiaca & - & 3 \\
$\begin{array}{l}\text { Morte súbita } \\
\text { Acidente vascular cerebral }\end{array}$ & -1 & 1 \\
$\begin{array}{l}\text { Endocardite infecciosa } \\
\text { TOTAL }\end{array}$ & 1 & 1 \\
\hline
\end{tabular}

GRÁFICO 1

CURVAS ATUARIAIS DE SOBREVIDA A LONGO PRAZO NOS 2 GRUPOS

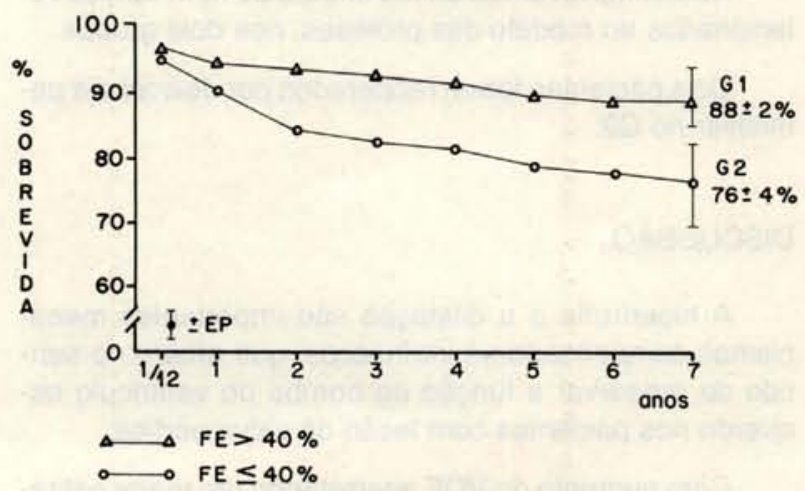

portanto, nítido predomínio no Grupo G2 de depressão da contratilidade $(20 \%)$, contra $5 \%$ no G1, no pós-operatório imediato. Não houve óbito trans-operatório em ambos os grupos.

As causas dos óbitos imediatos estão na Tabela 4 , sendo nítido o predomínio de insuficiência cardíaca no Grupo G2, o mesmo ocorrendo ao exame da Tabela 5 , que explicita as causas dos óbitos tardios.

A sobrevida, em sete anos, comparada está no Gráfico 1 , com $52 \%$ dos pacientes com quatro anos de seguimento completo e demonstra que há significância $p<$ 0,03 ente G1 e G2, mesmo considerando dois erros-padrão da média. A evolução clínica tardia está exposta, 
BARBOSA, G. V.; SOUZA, B. F.; REY, N. A.; WENDER, O. C.; PIMENTEL FILHO, P.; VIEIRA, S. R. R.; WOLMEISTER, H.; MANFROI, W. C.; WESTPHALEN, P. P. - Substituiçāo isolada da valva aórtica em pacientes com funçāo ventricular deprimida. Rev. Bras. Cir. Cardiovasc., 4(3): 195-201, 1989.

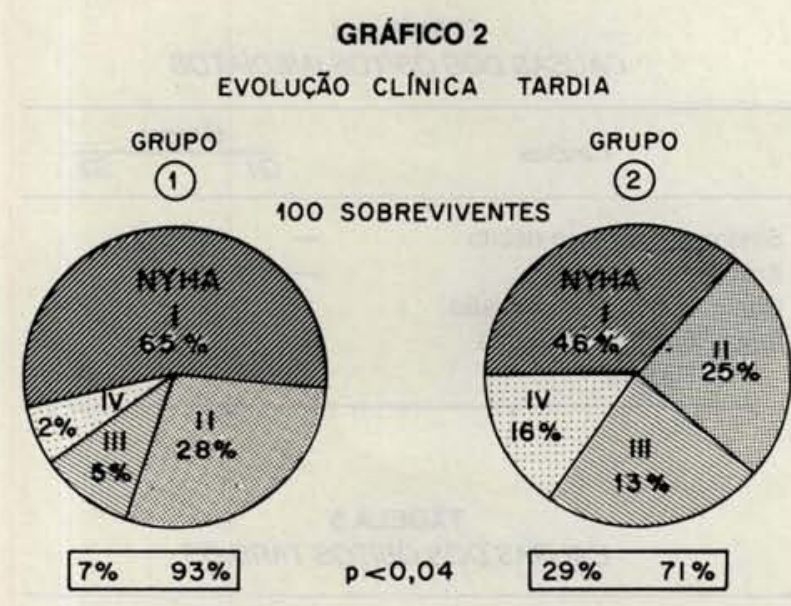

comparativamente, entre os grupos, no Gráfico 2, para 100 sobreviventes.

No grupo com FE > $(40 \%) 93 \%$ dos pacientes estão nas classes funcionais I e II da NYHA e somente $7 \%$ nas classes III e IV. No grupo com FE $\leqq(40 \%), 71 \%$ estão nas classes I e II e $29 \%$ nas III e IV, p $<0,04$.

No Grupo G2, $83 \%$ dos pacientes tomam digital e diuréticos e $38 \%$ vasodilatadores e/ou antiarrítmicos, contra $41 \%$ no Grupo G1.

Não comprovamos óbitos imediatos nem tardios relacionados ao modelo das próteses, nos dois grupos.

Dois pacientes foram reoperados por deiscência perivalvar no G2.

\section{DISCUSSÃO}

A hipertrofia e a dilatação são importantes mecanismos compensadores intrínsecos, que atuam no sentido de preservar a função de bomba do ventrículo esquerdo nos pacientes com lesão da valva aórtica.

Com aumento do VDF acarretando um maior estiramento da fibra cardiaca, a função de bomba ventricular é, incialmente, preservada, às custas de maior dispêndio de energia utilizada na contração pelo aumento da tensão da parede ventricular ${ }^{3}$, 5 . Neste estágio, a FE e o volume sistólico (VS) permanecem, ainda, dentro dos limites normais, apesar da função contrátil da fibra cardiaca já estar comprometida.

O aumento do VDF promove um aumento no tamanho dos sarcômeros e a manutenção da sobrecarga de volume o leva a não responder mais à lei de FrankStarling. Então, o mecanismo compensador falha, advindo aumento da pressāo diastólica final $(\mathrm{Pd} 2)$ e queda da fração de ejeção ${ }^{15,25}$.

O miocárdio distendido altera sua estrutura, ocorrendo deslizamento de células e distenção dos elemen- tos colágenos. Isto provoca dano celular, como necrose e fibrose disseminadas, afastamento entre as fibras miocárdicas, pois a alta compressão extravascular do miocárdio e a baixa perfusão coronária diastólica constituem-se numa combinação desfavorável para a perfusão subendocárdica ${ }^{27,} 32$.

Portanto, a depressão do inotropismo começa muito antes do aparecimento dos primeios sintomas e sinais de insuficiência cardiaca, mascarados pelos mecanismos compensadores. A FEVE em repouso pode ser facilmente obtida pela análise da ventriculografia pré-operatória em OAD. Tem sido utilizada como importante indicador de atividade cardíaca global e um índice fiel da extensão de encurtamento da fibra miocárdica. Apesar de certas limitaçōes, é considerada uma das melhores medidas da função de bomba do VE.

Vários autores demonstraram maior mortalidade global e persistência de limitação física aos exercícios, em pacientes com $\mathrm{FE}<40 \%{ }^{3.5}$. No Grupo G2, tivemos uma mortalidade global nitidamente superior ao G1 e uma sobrevida a longo prazo nitidamente inferior. A causa mais freqüente de óbitos no Grupo G2 durante os períodos imediato e tardio foi por insuficiência cardíaca, demonstrando que a extensão da doença miocárdica existente antes da operação é um fator relevante.

O dano miocárdico que se agrega durante a operaçăo, em forma de isquemia ou infarto trans-operatório traduzido pela ocorrência de síndrome do baixo débito, aparece na literatura numa freqüência de 5 a $10 \%{ }^{9}$, 24,33 .

Tivemos uma incidência de $5 \%$ no Grupo G1, sem óbito, e de $20 \%$ no G2, com três óbitos, e estes sobreviventes do Grupo G2 evoluíram para a NYHA III e IV no pós-operatório tardio. Isto demonstra a vulnerabilidade dos pacientes com função deprimida em agregar novos insultos ao miocárdio a despeito da proteção miocárdica, durante a cirurgia.

$\mathrm{Na}$ mortalidade tardia, predonimaram os eventos relacionados à depressão da contratilidade miocárdica. O Grupo G2 teve três óbitos adicionais, por insuficiência cardiaca refratária.

SANTINGA et alii ${ }^{29}$ enfatizam que o subendocárdio do VE é a região mais sujeita à isquemia, porque necessita receber todo o seu fluxo sangüíneo durante a diástole e, quanto mais hipertrofia concêntrica tiver, mais vulnerável se tornará.

A necrose subendocárdica é a causa de morte mais comum após a cirurgia tecnicamente com sucesso. Ela se instala sem obstruçāo anatômica das artérias coronárias, ocorrendo pela discrepância da oferta de oxigênio e a demanda durante a cirurgia é responsável, também, pela alta incidência de insuficiência cardíaca no pós-operatório e quando o paciente apresenta arritmia ventricular 
BARBOSA, G. V.; SOUZA, B. F.; REY, N. A.; WENDER, O. C.; PIMENTEL FILHO, P.; VIEIRA, S. R. R.; WOLMEISTER, H.; MANFROI, W. C.; WESTPHALEN, P. P. - Substituição isolada da valva aórtica em pacientes com função ventricular deprimida.

Rev. Bras. Cir. Cardiovasc., 4(3): 195-201, 1989.

tem alto risco de morte súbita; $a$ incidência, na literatura, vai de 4 a $38 \%$. Estes pacientes devem ser avaliados por Holter e o uso de drogas antiarrítmicas deve ser considerado. Tivemos um caso aos seis anos no G2 e aos 34 meses no G1.

A qualidade de vida é ditada pelo estado funcional do miocárdio ventricular esquerdo. Este fato fica demonstrado na análise da evolução clínica tardia do Gráfico 2 , onde os pacientes com $\mathrm{FE} \leqslant 40 \%$ levaram nítida desvantagem na regressão às classes I e II da NYHA. CLARK et alii ${ }^{9}$ realizaram troca da valva aórtica em 12 pacientes com IAO e fração de ejeção com média de $43 \%$, sem óbito operatório, mas com cinco óbitos tardios.

A função sistólica de bomba do VE melhorou em $50 \%$ dos pacientes, bem como regrediram moderadamente a dilatação e a hipertrofia de VE. A sobrevida atuarial em três anos foi de $61 \pm 15 \%$ e $90 \%$ dos sobreviventes estão na NYHA I e II. Houve leve aumento da FE de $43 \%$ para $49 \%$ no pós-operatório imediato.

Quando a regurgitação é suprimida, o volume sistólico (VS) retorna ao normal, propiciando que as fibras musculares circunferenciais preponderem, novamente, sobre as longitudinais, durante a contração, e o VE vai reassumindo a sua geometria normal.

A remoção da pós-carga excessiva pela substituição valvar permite a melhora imediata da ejeçāo ventricular esquerda, aumentando o volume minuto e diminuindo o consumo de oxigênio pelo miocárdio ${ }^{26,34}$.

O maior problema na avaliação dos efeitos da substiuição da valva aórtica na FVE é a dificuldade em separar os efeitos da remoção das condiçōes anormais de sobrecarga daqueles devido à depressão primária do miocárdio ${ }^{2}$.

Um fato relevante emerge, quando examinamos o Gráfico 3, que demonstra uma sobrevida melhor no Gru-

\section{GRÁFICO 3}

CURVAS ATUARIAIS DE SOBREVIDA A LONGO PRAZO COMPARATIVAS ENTRE DUAS SÉRIES CLIINICAS COM O GRUPO G 2

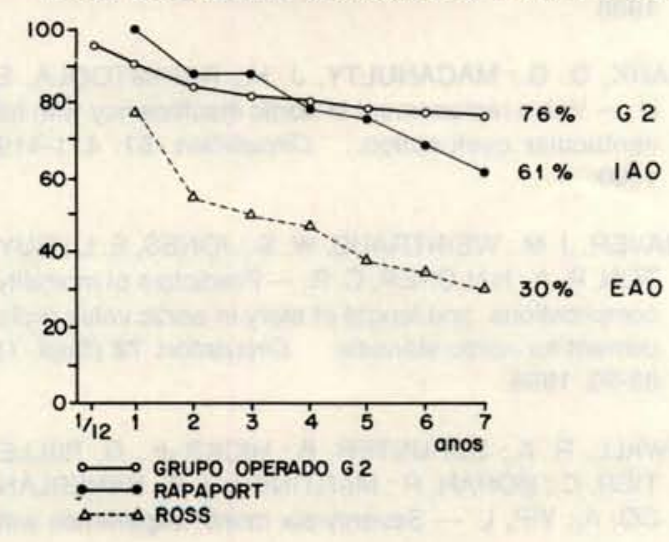

po com $\mathrm{FE} \leqq(40 \%)$, quando comparado a duas séries de pacientes tratados clinicamente.

O diâmetro médio da valva aórtica humana é de $24 \mathrm{~mm}$ no homem e $22 \mathrm{~mm}$ na mulher, correspondendo a uma área de fluxo de $5 \mathrm{~cm}^{2} \mathrm{e} 4 \mathrm{~cm}^{2}$, respectivamente.

STAREK et alii ${ }^{32}$ e DeWALL et alii ${ }^{11}$, realizaram avaliação hemodinâmica pós-operatória nos pacientes com próteses aórticas Meditronic-Hall e Omniscience. Utilizando a equação de Gorlin, encontraram área útil de fluxo de $2,17 \mathrm{~cm}^{2}$ no tamanho 23 até $3,09 \mathrm{~cm}^{2}$, no tamanho 29 , ficando claro que, na melhor das hipóteses, correspondem a $55 \%$ da área da valva humana. Esta estenose relativa pode ser responsável pela não regressão da dilatação ou hipertrofia cardiacas, no pósoperatório, impossibilitando a melhora significativa da funçāo ventricular ${ }^{26}$.

TURINA et alii ${ }^{33}$ relataram melhores resultados na IAO, quando a cirurgia foi indicada mais precocemente. A NYHA média foi de 2,1 e a FE $=58 \%$, a mortalidade operatória foi de $2 \%$, e a sobrevida em cinco anos de $90,6 \%$.

ISKANDRIAN et alii ${ }^{22}$ e DILWORT et alii ${ }^{12}$ demonstraram o valor da avaliação da FE pela ventriculografia radioisotópica, demonstrando a acuidade do método não invasivo em diagnosticar a queda da FE em exercício.

As próteses modernas têm baixo índice de complicações fatais e bom desempenho hemodinãmico ${ }^{10,25,29}$ e tornam-se, por isso, uma alternativa atraente para a cirurgia em fase mais precoce, considerando-se os índices de mortalidade e as complicações clínicas graves nos pacientes operados em classes funcionais avançadas.

\section{CONCLUSŌES}

1) Os pacientes com FEVE $\leqslant 40 \%$ comparados aos pacientes com FEVE > têm:

- Mortalidade global mais elevada $(p<0,05)$

- Menor sobrevida a longo prazo $(p<0,03)$

- Resultados clínicos menos satisfatórios ( $p<$ $0,04)$

2) Para obtermos melhores resultados é necessário indicar a cirurgia:

- Antes que a FEVE em repouso se mostre deprimida

- Sempre que em exercício baixe, independentemente da classe funcional do paciente

- Antes que se estabeleça intolerância aos exercicios de moderada intensidade. 
BARBOSA, G. V.; SOUZA, B. F.; REY, N. A.; WENDER, O. C.; PIMENTEL FILHO, P.; VIEIRA, S. R. R.; WOLMEISTER, H.; MANFROI, W. C.; WESTPHALEN, P. P. - Substituição isolada da valva aórtica em pacientes com função ventricular deprimida.

Rev. Bras. Cir. Cardiovasc., 4(3): 195-201, 1989.

RBCCV $44205-93$

BARBOSA, G. V.; SOUZA, B. F.; REY, N. A.; WENDER, O. C.; PIMENTEL FILHO, P.; VIEIRA, S. R. R.; WOLMEISTERN, H.; MANFROI, W. C.; WESTPHALEN, P. P. - Aortic valve replacement in patients with depressed left ventricular ejection fraction. Rev. Bras. Cir. Cardiovasc., 4(3): 195-201, 1989.

ABSTRACT: To evaluate the prognostic value of resting left ventricular ejection fraction (LVEF) a series of 210 consecutive patients with aortic valve disease operated upon between May 1981 and October 1988 was studied retrospectively. All patients had their aortic valve replaced by Omniscience or Meditronic prosthesis; 112 formed the study group because they have complete follow up and cineangiocardiographic data. Based on pre-operatory left ventricular ejection fraction the patients were divided in two groups: Group $1=52$ patients with LVEF $>40 \%, 39 \pm 12$ years, NYHA functional class 2.8 , and EF $58 \pm 10 \%$. Group $2=60$ patients with LVEF $\leqslant 40,48 \pm 17$ years, NYHA functional class 3.6 , and LVEF of $27 \pm 12 \%$. During surgery moderate hypothermia and hemodilution were utilized, with dubble oxygenator and cardioplegia with St. Thomas cardioplegic solution. Mean extracorporeal circulation and cardiac arrest time were respectively $82 \pm 18$ and $49 \pm 7$ minutes in Group 1 and $96 \pm 11$ and $55 \pm 6$ minutes in Group 2. The size of the prosthesis were $25.2 \pm 1.8 \mathrm{~mm}$ in Group 1. The short term mortality was $3.8 \%$ in Group 1 and 5.8 in Group 2. The long-term mortality was $4.0 \%$ in Group 1 and $8.7 \%$ in Group 2. In Group 1 there was a significant association between mortality and left ventricular systolic function. In the follow-up evaluation, among patients in Group 1, $65 \%$ were in class I, $28 \%$ were in functional class II, $5 \%$ in class III, and $2 \%$ in class IV. Among patients in group II $46 \%$ were in class I, $25 \%$ in class II, $13 \%$ in class III, and $16 \%$ in class IV. The 7 year survival rate was $88 \pm 2 \%$ in Group 1 and $76 \pm 4 \%$ in Group 2. Thus, patients with LVEF less than $40 \%$ had higher short and longterm mortality and worse clinical course. For better surgical results patients with aortic valve disease should be operated upon before the reduction of left ventricular systolic function.

DESCRIPTORS: ventricular function; heart valves, aortic, surgery.

\section{REFERÊNCIAS BIBLIOGRÁFICAS}

1 ADAMS, P. X.; CUNNINGHAM, J. N.; ITRELANIN, K.; BRAZIER, J. R.; REED, G. E.; SPENCER, F. C. Clinical experience using potassium induced cardioplegia with hipothermia in aortic valve replacement. J. Thorac. Cardiovasc. Surg., 75: 564-568, 1978.

2 ANDERSON, R. \& CRUMBLEY, A. J. - Ventricular function in surgical patients. Ann. Thorac. Surg., 35: 1-2, 1983.

3 BONOW, R. O.; DODD, J. T.; MARON, B. J.; O'GARA, P. T.; WHITE, G. G.; MCINTOSH, C. L.; CLARK, R. E.; EPSTEIN, S. E. - Long-term serial changes in left ventricular function and reversal of ventricular dilatation after valve replacement for chronic aortic regurgitation. Circulation, 78: 1108-1120, 1988.

4 BONOW, R. O.; ROSING, D. R.; MARON, B. J.; MCINTOSH, C. L.; JOWES, M.; BACHARACH, S. L.; GREEN, M. V.; CLARK, R. E.; EPSTEIN, S. E. - Reversal of left ventriculr dysfunction after aortic valve replacement for chronic aortic regurgitation: influence of duration of preoperative left ventricular dysfunction. Circulation, 70: 570-579, 1984.

5 BORER, J. S.; ROSING, D. R.; KENT, K. M.; BACHARACH, S. L.; GREEN, M. V.; MCINTOSH, C. J.; MORROW, A. G.; EPSTEIN, S. E. - Left ventricular function at rest and during exercise after aortic valve replacement in patients with aortic regurgitation. $A m$. J. Cardiol., 44: 1297-1305, 1979.
6 CARABELLO, B. A.; WILLIAMS, H.; GASH, A.; KENT, R.; BELBER, D.; MAURER, A.; SIEGEL, J.; BLASIUS, K.; SPANN, J. F. - Hemodynamic predictors of outcome in patients undergoing valve replacement. Circulation, 74: 1309-1316, 1986.

7 CHRISTAKIS, G.; WEISEL, R. D.; DAVID, T. E.; SALERNO, T. A.; IVANOV, J. - Predictors of operative survival after valve replacement. Circulation, 78 (Supl. 1): 25-34, 1988.

8 CHRISTAKIS, G.; WEISEL, R. D.; FREMES, S. E.; TEOH, K. H.; SKALENDA, J. P.; TONG, C. P.; AZUMA, J. Y.; SCHWARTZ, L.; MICKLEBOROUEH, L. L.; SCULLY, H.; GOLDMAN, B. S.; BAIRD, R. J. - Can the results of contemporary aortic valve replacement be improved? J. Thorac. Cardiovasc. Surg., 92: 37-46, 1986.

9 CLARK, D. G.; MACANULTY, J. H.; RAHIMTOOLA, S. H. - Valve replacement in aortic insufficiency with left ventricular dysfunction. Circulation, 61: 411-419, 1980.

10 CRAVER, J. M.; WEINTRAUB, W. S.; JONES, E. L.; GUYTON, R. A.; HALCHER, C. R. - Predictors of mortality, complications, and lenght of story in aortic valve replacement for aortic stenosis. Circulation, 78 (Supl. 1): $85-90,1988$.

11 DeWALL, R. A.; SCHUSTER, B.; HICKS Jr., G. PELLETIER, C.; BONAN, R.; MARTINEU, J. P.; PANEBLANCO, A.; YIP, L. - Seventy-six month-experience with 
BARBOSA, G. V.; SOUZA, B. F.; REY, N. A.; WENDER, O. C.; PIMENTEL FILHO, P.; VIEIRA, S. R. R.; WOLMEISTER, H.; MANFROI, W. C.; WESTPHALEN, P. P. - Substituição isolada da valva aórtica em pacientes com função ventricular deprimida. Rev. Bras. Cir. Cardiovasc., 4(3): 195-201, 1989.

the Omniscience cardiac valve. - J. Cardiovasc. Surg., 28: 328-332, 1987.

DILWORTH, L. R.; AISEN, A. M.; MANCINI, G. B. J.; LANDE, I.; BUDA, A.; MICH, A. A. - Determination of left ventricular volumes and ejection fraction by nuclear magnetic resonance imaging. $A m$. Heart J., , 112: 24-32, 1987.

DODGE, H. T.; SANDLER, H.; BALLEW, D. W.; LORD Jr., J. D. - The use of byplane angiocardiography for the measurement of left ventricular volume in man. Am. Heart J., 60: 762-778, 1960.

14 DUBROFF, J. M.; CLARK, M. B.; WONG, C. Y. H.; SPOTNITZ, A. J.; COLLINS, R. H.; SPOTNITZ, H. M. Left ventricular ejection fraction during cardiac surgery: a two-dimensional echocardiographic study. Circulation, 68: 95-103, 1983.

15 FISCHL, S. J.; GORLIN, R.; HERMAN, M. V. - Cardiac shape and function in aortic valve disease: physiologic and clinical implications. Am. J. Cardiol., 39:170-176, 1977.

16 FORMAN, R.; FIRTH, B. G.; BARNARD, M. S. - Prognostic significance of preoperative left ventricular ejection fraction and valve lesion in patients with aortic valve replacement. Am. J. Cardiol., 45: 1120-1125, 1980.

17 GAASCHIW, W. H.; ANDRIAS, C. W.; LEVINE, H. J. Chronic aortic regurgitation: the effect of aortic valve replacement on left ventricular volume, mass and function. Circulation, 58: 825-836, 1978.

18 GAULT, J. H.; COVELL, J. W.; BRAUNWALD, E.; ROSS Jr., J. - Left ventricular performance following correction of free aortic regurgitation. Circulation, 42 : 773-784, 1970.

19 GREENE, D. G.; CARLISLE, R.; GRANT, C.; BUNNELL, I. L. - Estimation of left ventricular volume by oneplane cineangiography. Circulation, 35:61-74, 1967.

20 GRUNKMEIER, G. L. \& STARR, A. - Actuarial analysis of surgical results: rationale and method. Ann. Thorac. Surg., 24: 404-413, 1977.

21 HILDNER, F. J.; JAVIER, R. P.; COHEN, L. S.; SAMET, P.; NATHAN, M. J.; YAHR, W. Z.; GREENBERG, J. J. - Myocardial dysfunction associated with valvular heart disease. Am. J. Cardiol., 30: 319-328, 1972.

22 ISKANDRIAN, A. S.; HEO, J.; PA, P. - Radionuclide angiopgrahic evaluation of left ventricular performance at rest and during exercise in patients with aortic regurgitation. Am. Heart J., 111: 1143-1147, 1986.

23. KENNEDY, J. W.; TRENHOLME, S. E.; KASSER, I. S - Experience and laboratory reports: left ventricular volume and mass from single-plane cineangiocardiogram: a comparison of anteroposterior and right ante- rior oblique methods. Am. Heart J., 80: 343-357, 1970.

24 LYTLE, B. W.; COSGROVE, D. M.; GILL, C. C.; TAYLOR, P. C.; STEWART, R. W.; GOLDING, L. A.; GOORMASTIC, M.; LOOP, F. D. - Aortic valve replacement combined with myocardial revascularization. I Thorac. Cardiovasc. Surg., 95: 402-414, 1988.

25 MEHMEL, H. C.; OLSHAUSEM, K.; SCHULER, G.; SCHWARZ, F.; KUBLER, W. - Estimation of left ventricular myocardial function by the ejection fraction in isolated, chronic, pure aortic regurgitation. Am. J. Cardiol., 54: 610-616, 1984.

26 MONRAD, E. S.; HESS, O. M.; MURAKAMI, T.; MONOGI, M. D.; CORIN, W. J.; KRAYENBUEHL, H. P. - Abnormal exercise hemodynamics in patients with normal systolic function late after aortic valve replacement. Circulation, 77: 613-624, 1988.

27 PEIXOTO, E. S. C.; BARROS FILHO, C. M.; PONTES, A. C. P. S.; LEITE, A. A.; BRITO, A. H. X.; DOHMANN, H. J. F.; ABRĀO, C.; OLIVEIRA, P. S.; AMIND, J. G. C.; STUDART, P. C. C. - Correlação entre a clínica do paciente avaliada pela classe funcional e a função ventricular esquerda na insuficiência aórtica crônica: II. Estresses. Arq. Bras. Cardiol., 51: 231-237, 1988.

28 SALOMON, N. W.; STINSON, E. B.; OYER, P.; COPELAND, J. G.; SHUMWAY, N. E. - Operative treatment of congenital aortic stenosis. Ann. Thorac. Surg., 26: 452-460, 1978.

29 SANTINGA, J. T.; KIRSH, M. M.; BRADY, T. J.; THRALL, J.; PITT, B. - Left ventricular function in patients with ventricular arrhythmias and aortic valve disease. Ann. Thorac. Surg., 35: 152-154, 1983.

30 SCOTT, W.; MILLER, C.; HAVERICH, A.; DAWKINS, L.; MITCHELL, R. S.; JAMIESON, S. W.; OYER, P. E.; STINSON, E. B.; BALDWIN, J. C.; SHUMWAY, N. Determinations of operative mortality for patients undergoing aortic valve replacement. J. Thorac. Cardiovasc. Surg., 89: 400-413, 1985.

31 SCHWARZ, F.; FLAMENG, W.; SCHAPER, J.; LANGEBARTELS, F.; SESTO, M.; HEHRLEIN, F.; SCHLEPPER, M. - Myocardial structure and function in patients with aortic valve disease and their relation to postoperative results. Am. J. Cardiol., 41: 661-669, 1978.

32 STAREK, P. J. K.; BEAUDET, R.; HALL, K. - The Medtronic-Hall valve: development and clinical experience. Card. Surg., 1: 223-236, 1987.

33 TURINA, J.; TURINA, M.; ROTHLIN, M.; KRAYENBUEHL, H. P. - Improved late survival in patient with chronic aortic regurgitation by earlier operation. Circulation, 70 (Supl. 1): 147, 1984.

34 WISENBAUGH, T.; BOOTH, D.; DEMARIA, A.; MISSEN, S.; WATERS, J. - Relationship of contractile state to ejection performance in patients with chronic aortic valve disease. Circulation, 73: 47-53, 1986. 